\title{
El Psicólogo Educativo en Europa
}

\section{The Educational Psychologist in Europe}

\author{
José A. León \\ Universidad Autónoma de Madrid, España
}

\begin{abstract}
Resumen. Europa cuenta con extraordinarios e ilustres psicólogos que fomentaron la educación como un aspecto esencial de la Psicología desde sus inicios. Aunque hubo siempre una necesidad y justificación por desarrollar la figura del psicólogo educativo, ésta se desarrolló de manera particular en cada país de origen adaptándose a sus propias necesidades, por lo que el desarrollo de la Psicología fue desigual en cada uno de los países. La situación actual hace que aspectos nucleares del psicólogo como es su identidad, formación, funciones y profesionalización sean aún muy dispares en los países de nuestro entorno, por lo que se requiere de una integración y una redefinición que haga causa común de las funciones, formación y especialización y profesionalización común en todos los países de la unión. En este artículo se analiza la situación inicial y actual del psicólogo educativo en varios países europeos y se proponen algunas de las tareas pendientes e iniciativas que inciden en un marco común del psicólogo educativo en Europa.

Palabras clave: psicología educativa, psicólogo educativo, marco común del psicólogo educativo en Europa, EuroPsy, ESPIL 2010, Federación Europea de Asociaciones de Psicólogos, EFPA.
\end{abstract}

\begin{abstract}
Europe has extraordinary and distinguished psychologists who have promoted education as an essential aspect of psychology since its inception. Although there has always been a need and justification to further develop the figure of the educational psychologist, it in fact developed in a particular way in each country and in a way adapted to their own needs. This has caused the development of psychology to be uneven in each country. The current situation means that the central issues facing psychologists, such as is their identity, training, and professional roles, still vary widely between European countries. Integration is therefore required to find agreement on the redefinition of the roles, training, professionalization and specialization in all countries of the European Union. This paper analyzes the initial and current situation of educational psychologists in several European countries and suggests some of the remaining tasks and initiatives that would affect a common framework for the educational psychologist in Europe.

Keywords: common framework of the educational psychologist in Europe, Educational psychology, educational psychologist, EuroPsy, ESPIL 2010, European Federation of Psychologists' Associations, EFPA.
\end{abstract}

Europa cuenta con un importante grupo de ilustres psicólogos que fomentaron la educación como un aspecto esencial de la Psicología desde sus inicios en sus respectivos países. Aunque hubo siempre

La correspondencia sobre este artículo debe enviarse al autor al Departamento de Psicología Básica. Facultad de Psicología. Universidad Autónoma de Madrid. Ivan Pavlov, 6. Ciudad Universitaria de Cantoblanco. 28049 Madrid. E-mail: Joseantonio. leon@uam.es una necesidad y justificación por desarrollar la figura del psicólogo educativo, ésta se desarrolló de manera particular en cada país de origen adaptándose a sus propias necesidades, a su propio ritmo, por lo que el desarrollo de la Psicología Educativa fue desigual entre los países europeos. Por razones obvias no nos centraremos aquí en la Psicología 
española, puesto que este tema se ha abordado en artículos precedentes (véase, por ejemplo, Carpintero, 2011; Fernández Barroso, 2011; Méndez, 2011 y Pérez Solís, 2011, todos en este mismo número). Podemos señalar que durante el primer tramo del siglo veinte la Psicología Educativa europea intenta responder en la medida de sus posibilidades a los retos que cada país tiene y muy especialmente tras finalizar la Segunda Guerra Mundial. A partir de este momento hay muchos y serios desarrollos de la Psicología Educativa que se incrementan en las décadas de los sesenta a los noventa con aportaciones muy interesantes y novedosas. Pero ello conlleva que en la situación actual haya aspectos nucleares del psicólogo como es su identidad y funciones atribuibles dentro del sistema educativo, como también es su formación, especialización y profesionalización que, al seguir siendo muy dispares entre los países de nuestro entorno, dificulta enormemente una puesta en común de objetivos, del establecimiento de una imagen unitaria del psicólogo educativo en el que se determinen sus funciones más básicas, de una movilidad laboral reducida o de una formación especializada más común y compartida.

Las reformas educativas que actualmente se vienen llevando a cabo en los diversos países de la Unión respecto a los estudios universitarios como es la obtención del grado y de posgrado, ayudan poco o nada a mejorar la situación actual. El hecho, por ejemplo, de que en algunos países se requiera de tres años para obtener el grado de psicología mientras que en otros sea, como en España, de cuatro años y que los másteres que se impartan sean de 60 ó 90 créditos, no hace más que constatar las diferencias desde las que se partían en el pasado y no confluyen en una Europa más integradora. Incluso dentro de un mismo país, las ofertas son muy distintas y dispares y llevan a una mayor confusión del papel del psicólogo educativo dentro del sistema educativo propio. En España, por ejemplo, este aspecto resulta flagrante. Existe, por ejemplo, un máster oficial y obligatorio del Profesorado que se imparte en las facultades de educación y que prepa- ra para la docencia en la etapa de Educación Secundaria (Especialidad Orientación Educativa), lo que otorga el papel de orientador a quien lo cursa y requiriéndose para optar a plazas públicas y privadas relativas al profesorado y a la orientación en los centros escolares. Por su parte, algunas facultades de Psicología españolas optan por ofrecer un máster en Psicología de la Educación, de carácter genérico y conceptual y cuya obtención faculta a cualquier profesional de la educación a ejercer como "pseudopsicólogo educativo" (véase a este respecto Pérez Solís, 2011, en este mismo número; también Méndez, 2011). Estos másteres, además, no cuentan con las asociaciones profesionales como es el Colegio Oficial de Psicólogos (COP), sino que su diseño y desarrollo se han realizado sólo desde las universidades, lo que dificulta una visión conjunta y coordinada con la profesión y el propio COP. Hay, por último, otras propuestas que se están abordando también desde otras posiciones dentro de las facultades de Psicología que, junto con el COP, tratan de diseñar másteres específicos para el psicólogo educativo (véase Pérez Solís, 2011, en este mismo número). Esta última propuesta está en consonancia con los retos y propuestas europeas para el siglo XXI desde donde se plantea la necesidad de desarrollar una Psicología Educativa común entre los países miembros de la Unión Europea que permita una formación unitaria y común, una movilidad y una especialización profesional entre todos los países de la Unión.

En este artículo se analiza la situación inicial y actual del psicólogo educativo tomando cuatro instantáneas de cuatro de los países europeos más relevantes como son Francia, Alemania, Reino Unido y Finlandia, donde se pueden constatar estas diferencias a las que nos referíamos en los párrafos anteriores para plasmar, en una segunda parte, algunos datos que muestran la situación actual del psicólogo educativo en toda Europa. Finalmente, en una tercera parte se proponen algunas tareas pendientes e iniciativas que suponen los retos de futuro sobre la creación de un marco común del psicólogo educativo en Europa y a los que debemos y podemos dar respues- 
ta. Sobre estas iniciativas sobresalen la necesidad de una integración y una redefinición que haga causa común de las funciones, formación y especialización y profesionalización común en todos los países de la Unión Europea.

\section{Cuatro instantáneas sobre los inicios a la situación actual de la Psicología Educativa en Europa}

Puesto que no nos es posible por razones de espacio tratar aquí la evolución de la Psicología reciente en todos los países europeos, hemos optado por representar, junto a la ya descrita situación española en otros trabajos precedentes, países que fueron claves en el desarrollo de la Psicología Educativa en Europa y que reflejan las divergencias y múltiples puntos de vista de la Psicología. Algunos de estos países poseen mucha carga histórica y mucha presencia en la Psicología Educativa como son Francia y Reino Unido. Otros, en cambio, tienen una posición dominante, bien por su situación económica como es el caso de Alemania y que tuvo, además, la particularidad de haber vivido dos Psicologías Educativas diferentes, una a cada lado del "muro", o bien por su situación de su modelo educativo, considerado actualmente de los mejores del mundo, como es el caso de Finlandia. Analizaremos de modo necesariamente breve cada uno de ellos.

\section{La Psicología Educativa en Francia}

Francia fue, en realidad, uno de los primeros países que se interesó por introducir la Psicología en las escuelas. La historia reciente se inicia en 1894 con Alfred Binet, quien publica su primer artículo sobre la Psicología en la escuela primaria (Binet y Vaschide, 1897). Binet, junto con Pierre Vaney, también creó el primer laboratorio de psicología y pedagogía en un centro escolar parisino en 1899. En 1905, cuando creó el primer test de inteligencia en París, su prueba Binet-Simon, estaba interesado en los niños con necesidades educativas especiales. La prueba fue aplicada con el objeto de diagnosticar retraso intelectual infantil y derivar los casos detectados a centros donde hubiese educación especial. Para descubrir cuáles eran sus necesidades, necesitaba tener una idea de cuál era la capacidad media. Quizás por todo ello se le considera el padre de la psicología educativa. A estas iniciativas se unió, en 1909, Marcel Foucault, quien abrió un laboratorio de psicología escolar en Montpellier, coincidiendo en ese mismo año el primer servicio de orientación vocacional (Foucault, 1923).

Si bien estas iniciativas fueron más personales que institucionales, no fue hasta el final de la Segunda Guerra Mundial cuando surge la necesidad de preparar psicólogos cualificados para formar parte de los servicios escolares de Francia. Así, surge el primer psicólogo educativo de la postguerra, Bernard Andrey en 1945 y los primeros trabajos de Henry Wallon (1869-1962) en 1947 siendo, a partir de aquí, donde el propio Ministerio de Educación francés asume formar psicólogos educativos con el objetivo prioritario de identificar y diagnosticar a aquellos niños que necesitasen de una educación especial. Ello requirió del desarrollo de pruebas y test psicométricos para la evaluación, y de teorías sobre psicopatología para su diagnóstico. Si bien esta tendencia de evaluar la inteligencia se desarrolló durante los años cincuenta, esta concepción se fue modificando paulatinamente durante los años 60 hacia el fracaso escolar, dado que en aquella época afectaba a más de la mitad de los niños entre 6 y 12 años. La concepción educativa general se basó en la idea de que el fracaso escolar se abordaba con mayor eficacia desde la prevención que desde la intervención. Así, sobre esta base se crearon en 1970 los equipos de ayuda psicopedagógica (Groupes d'Aide Psycho-Pédagogique) que se centraron durante 20 años en la prevención del fracaso escolar en la educación primaria, obteniendo resultados dispares. Por un lado, obtuvieron réditos importantes en la mejora de la conducta emocional y en la integración del estudiante en el aula, pero no consiguieron el éxito 
deseado respecto a áreas relacionadas con su principal misión, la de mejorar las habilidades de aprendizaje de los escolares y reducir así los niveles de fracaso escolar. Ante estos resultados, no del todo satisfactorios, estos equipos fueron reemplazados por otros, The Réseau d'Aides Spécialisées aux Eléves en Difficulté, y cuya composición estaba formada por un psicólogo educativo y dos profesores de educación especial. Entre sus objetivos se incluyeron las acciones preventivas dentro de los centros escolares junto con la incorporación paulatina de tareas que incrementasen la inclusión educativa de todos los niños con necesidades educativas especiales. Un dato importante que ocurrió en 1985 fue el reconocimiento del título de psicólogo educativo promovido por la Asociación de Psicólogos Educativos Franceses (Guillemard, 2007). La titulación y profesionalización del psicólogo educativo quedó así legalmente reconocida. Existen, actualmente en Francia, tres tipos de psicólogos educativos. Por un lado está la figura del psicólogo escolar que trabaja en los centros de educación primaria y pública (alrededor de 3000). Por otro, predomina el psicólogo como orientador vocacional en centros de enseñanza secundaria y universidad también públicos (alrededor de 4000). Y tercero, el psicólogo educativo que trabaja en centros privados (más minoritario).

En Francia, y a diferencia de otros países de su entorno, existe un especial interés por desarrollar una infraestructura sólida del psicólogo educativo, tanto para crear asociaciones que reivindican la profesionalización del psicólogo educativo, como por difundir y editar revistas especializadas en educación y profesión. Así, hay actualmente tres asociaciones de psicólogos educativos: la Asociación Francesa de Psicólogos Educativos (Association Française des Psychologues Scolaires) responsable de la publicación cuatrimestral de Psychologie \& Education y del periódico Echanges. Esta asociación fue la responsable de editar el primer manual en francés en el que se describe la organización y las prácticas de los psicólogos educativos (Guillemard y Guillard, 1997). Otra agrupación es la Asociación de Psicólogos Orientadores Vocacionales (The
Association des Conseillers d'OrientationPsychologues de France) que publica la revista Questions d'Orientation y también un periódico, $\mathrm{La}$ Lettre d l'ACOPE. La tercera es la Asociación Nacional de Psicólogos de la Enseñanza Católica y es de carácter privado (Association Nationale des Psychologues de 1'Enseignement Catholique). Además de las tres citadas existe otra sociedad más antigua como es la Sociedad Francesa de Psicología (Societé Française de Psychologie) y publica dos revistas cuatrimestrales, Psychologie Française y Pratiques Psychologiques.

La propuesta de formación del psicólogo educativo que se realiza en Francia es muy singular y especializada. Se inicia con la obtención del grado que ya parte de dos vertientes: Una se dirige a la formación especializada del psicólogo educativo (o psicólogo escolar) en centros de educación primaria y otra vertiente se dirige hacia el psicólogo educativo que se especializa en orientación vocacional y que trabaja en los centros de secundaria. La formación de grado del psicólogo educativo (o psicólogo escolar) consiste en un total de 300 horas. Entre los contenidos que se imparten figuran, entre otros, psicología y aprendizaje social, desarrollo socio-emocional y socio-cognitivo del niño, funcionamiento emocional en situaciones de la vida real, teorías y métodos de evaluación psicológica, tanto individual como en grupo dentro del aula, Psicología y Sociología de las relaciones en grupos y organizaciones, ergonomía escolar y ética profesional. Posteriormente, se realiza un máster en un servicio psicológico bajo la supervisión de un psicólogo educativo. Las tareas asumidas por este colectivo de psicólogos, junto al resto del equipo multiprofesional y de sus relaciones con los profesores y familias, se dirigen hacia la prevención de dificultades escolares, contribución en el diseño y desarrollo de proyectos escolares, concepción, desarrollo y evaluación de programas de apoyo individual y colectivo para alumnos con dificultades de aprendizaje y de conducta, y contribución a la integración de niños con hándicap en escuelas regulares. Para trabajar en los centros públicos de primaria la formación se 
completa, además, con una formación de dos años de profesor de primaria y se concluye al completar una experiencia docente de un mínimo de tres años.

Por el contrario, para alcanzar la especialización de orientación vocacional debe recibir un curso de formación especializada de dos años para acreditarse como psicólogo (aunque en este caso no se requiere ser profesor para trabajar en los centros privados). En estos casos, además de la formación universitaria de grado, tienen que realizar un máster de dos años en psicología educativa. Así, la formación del psicólogo especializado en orientación vocacional es de unas 500 horas en el que se incluyen los contenidos de teorías y aplicaciones de la psicología, teorías prácticas de metodología de orientación vocacional y escolar, teoría y práctica y metodología de la dirección de orientación hacia grupos y organizaciones (evaluación, comunicación y cooperación con las familias).

Como puede observarse en esta propuesta francesa, hay una sobre especialización del psicólogo educativo distinta para la educación primaria y secundaria, con roles, funciones y responsabilidades también diferentes. Existe, sin embargo, una tendencia e interés en la reunificación del psicólogo educativo de primaria y psicólogo de orientación vocacional de secundaria, como recoge Guillemard (2007), quien resume algunos de los hitos obtenidos en estos últimos años en la formación y profesionalización de los psicólogos educativos en Francia, como es el reconocimiento legal de la figura del psicólogo educativo, la determinación de ajustar la formación y la práctica a las necesidades reales y durante un periodo de formación universitaria de cinco años, la reducción progresiva de la influencia del psicoanálisis sobre la práctica profesional otorgando una mayor importancia a otras aproximaciones teóricas como es la psicología cognitiva, la neuropsicología, la organización ambiental o el decremento progresivo de la atención individualizada que se contrasta con el incremento en la planificación de proyectos y del trabajo organizacional y colectivo.

\section{La Psicología Educativa en Reino Unido (Escocia, Inglaterra y Gales)}

Es en el Reino Unido donde se emplean de manera más extendida los términos educational psychology y educational psychologist (psicología educativa y psicólogo educativo) en lugar de school psychology y school psychologist (psicología escolar y psicólogo escolar). Bajo estos dos términos se engloba y representa todo lo relacionado con la educación y la profesión.

El inicio de la Psicología Educativa en el Reino Unido estuvo muy vinculado al estudio de las diferencias individuales y la orientación infantil. Wooldridge (1994) detalla muy bien estos inicios que datan en 1860 (véase también para mayor información Squires y Farrell, 2007, o Topping, Smith, Barrow, Hannah y Kerr, 2007). La justificación de esta orientación por el estudio de las diferencias individuales y la orientación infantil viene como consecuencia de la implantación de la educación obligatoria que en el Reino Unido comienza en 1870. Ante esta situación, los profesores de la época se enfrentaron a un reto enorme al tener que enseñar a estudiantes con edades, rasgos, habilidades muy diferentes entre sí disponiendo, además, de muchos alumnos con dificultades de aprendizaje y con minusvalías severas añadidas. Conviene recalcar aquí que en este tiempo la Psicología se introducía como una asignatura universitaria con lo que aún no estaba desarrollada. Como forma de paliar este enorme problema se encargaba a los oficiales médicos la difícil tarea de decidir qué estudiante era adecuado para asistir a un centro escolar ordinario o a un colegio especial o si, simplemente, se le consideraba ineducable y no iba a la escuela. Como era esperable, este sistema tuvo muchas imperfecciones, puesto que la mayoría de los oficiales médicos no disponían de conocimientos psicológicos ni tampoco de herramientas necesarias para decidir de manera apropiada la ubicación óptima de cada niño. A esto se sumaban también las preocupaciones de los profesores acerca de dificultades y desajuste social, emocional o de conducta de sus estudiantes y la importancia de prevenirlos. 
En ese tiempo, Francis Galton (1822-1911) ya se interesaba por el estudio científico del niño, investigando las diferencias individuales de los alumnos mediante entrevistas que realizaba a padres y profesores. Partiendo de sus indagaciones asesoraba sobre la evaluación y el tratamiento. A Galton se le considera el «padre» de la Psicología Diferencial, al aplicar los principios de su primo, Darwin, al estudio de las diferencias individuales de las capacidades humanas, siempre desde una perspectiva adaptativa y biológica. En 1913, Cyril Burt, quien compartía el interés de Galton sobre las diferencias individuales y la naturaleza hereditaria de la inteligencia, llegó a ser el primer psicólogo profesional, contratado en aquel entonces por el Ayuntamiento de Londres. Él fue quien inició los estudios psicométricos para evaluar y categorizar las puntuaciones obtenidas de los niños. Poco más tarde, en Escocia (Glasgow y Edimburgo), surgieron grupos de especialistas clínicos-multiprofesionales de orientación infantil que, constituidos por psiquiatras, psicólogos educativos y trabajadores sociales, formaban a profesores especializados para abordar una educación especial para niños con hándicaps metales. Su trabajo también se extendía hacia problemas emocionales y de conducta, tratando de comprender y, posiblemente, también de prevenir, las conductas inadaptadas de los jóvenes. Actualmente, existen otros servicios de psicología educativa, como los que se ofrecen en Escocia, que tienen una base de elementos donde las claves sobre las que se desarrollan sus actividades se basan en trabajos de integración, en un intento por coordinar los diferentes servicios que trabajan con niños y adolescentes, estableciendo planes para tratar de minimizar su intrusión en la vida de los niños y de sus familias. También abordan la promoción de la inclusión social en la educación, siendo clave el papel de los psicólogos en este apartado. Resulta igualmente interesante el desarrollo de un servicio post escolar, donde se ayuda a los jóvenes más vulnerables en su transición desde la adolescencia hacia la edad adulta.

La Psicología en el Reino Unido está muy representada y difundida en revistas de prestigio interna- cional. La máxima representante de los psicólogos en el Reino Unido es la British Psychological Society (fundada en 1901) y que cuenta con 10 divisiones. Una de ellas es la Division of Educational and Child Psychology, que representa a los intereses profesionales dentro de la asociación principal, a la vez que promociona la Psicología Educativa a través de la distribución de información, mantenimiento de estándares profesionales, la formación de postgrado y la supervisión de psicólogos en prácticas. Existe también un sindicato para los psicólogos educativos, Association of Educational Psychologists, que vela por las condiciones laborales y económicas de los psicólogos. Las principales revistas del psicólogo educativo son: The British Journal of Educational Psychology (publicada por la British Psychological Society), Educational Psychology in Practice (Carfax Pubishing), Educational Psychology in Scotland y The Psychologist y Debate, todas ellas editadas por las diferentes Divisiones de la British Psychological Society. Esta asociación posee tambien mucha influencia sobre la formación profesional del psicólogo educativo. De hecho, es el organismo que acredita la formación especializada del psicólogo educativo y que consiste, una vez obtenido el título universitario en psicología, en un programa de dos años completos de formación profesional especializada. Hay, por tanto, una puesta muy fuerte hacia la profesionalización en las materias que integran la especialización. Por ejemplo, para la competencia práctica, se requiere de conocimientos precisos y rigurosos sobre intervenciones o acciones educativas hacia el niño, el aula, la familia, el centro escolar, la comunidad y el distrito escolar donde vive el alumno. Se fomenta la práctica científica, el ser autocríticos, conscientes y reflexivos en las prácticas que realizan. Estos programas de formación combinan el estudio académico con el aprendizaje práctico que se dirigen, a su vez, a promover el desarrollo de actitudes, habilidades y conocimientos en relación al desarrollo del niño, ya sea este desarrollo normal o excepcional, conocer bien los métodos de evaluación e intervención, ya sea individualizada o colectiva, así como el conoci- 
miento y uso de los distintos contextos en los que se desenvuelve la actividad educativa y saber utilizarlos. Especial atención se dedica también a la metodología de investigación y evaluación. Actualmente, existe una docena de programas de doctorado en psicología educativa que han sido acreditados por esta asociación.

La Bristish Psychological Society ha desarrollado también un currículo sobre la preparación del psicólogo educativo (Bristish Psychological Society Subject Benchmarks in Educational Psychology, 2005a). Los contenidos académicos incluyen materias nucleares tales como desarrollo evolutivo, psicología cognitiva, psicología social, y métodos de investigación. En él se describe un núcleo de competencias básicas que se requieren para el conocimiento, comprensión y habilidades profesionales de la práctica de la psicología educativa. Entre estas competencias se incluyen las siguientes:

- Promocionar la comunicación efectiva y las habilidades interpersonales (e.g., consultas, habilidades interpersonales y negociación dentro de un rango amplio de edades comprendidas entre jóvenes y adultos y ante una variedad de contextos, habilidades para gestionar y participar en reuniones, incluyendo la elaboración de presentaciones y el trabajo en equipo).

- Promover el desarrollo dentro de contextos (e.g., comprender el impacto de las barreras de aprendizaje mediante el análisis de una variedad amplia de diferentes contextos, conocimiento de la política local y nacional, de la legislación vigente).

- Facilitar el cambio a través de la evaluación e intervención (por ejemplo, los conocimientos y habilidades en la utilización de modelos para resolver problemas con un fuerte espíritu de colaboración para facilitar el cambio, con un conocimiento preciso de una gama de enfoques de evaluación e intervención y de su eficacia mediada por los contextos, así como una capacidad para recopilar y sintetizar la información de la evaluación de necesidades y de las intervenciones).
- Promover la investigación y la evaluación (e.g., potenciar las habilidades en la realización de investigación y utilizarlas de manera crítica para informar sobre la práctica basándose en la evidencia).

- Reconocer marcos para la práctica profesional (e.g., habilidades y marcos de trabajo dentro y fuera de distintos contextos, basándose en las teorías psicológicas y dentro de la legislación vigente).

- Preparar a los profesionales en prácticas para ser autónomos en la elaboración y presentación de elementos básicos.

\section{La Psicología Educativa en Alemania}

Aunque se atribuye a William Stern ser el primero en demandar la figura del psicólogo educativo en Alemania, durante su conferencia en Hamburgo en 1911, fue Hans Lämmermann a quien realmente se le consideró como el primer psicólogo educativo en Alemania, allá por el año 1922. La idea inicial de la que se partía en aquella época era introducir conocimientos y métodos que se impartían desde la Psicología académica universitaria directamente en la práctica de la enseñanza diaria. Dentro de las principales tareas que se le encomendaban al psicólogo estaba la de evaluar la habilidad e inteligencia como forma de orientar y facilitar a los estudiantes un aprendizaje óptimo. Sin embargo, poco después, con la llegada en 1933 del periodo nazi, el papel del psicólogo educativo quedó relegado, por lo que su desarrollo se detuvo durante la Segunda Guerra Mundial (véase Dunkel, 2007). Como es sabido, en 1945 el país fue dividido en Alemania Occidental, fuertemente influida por los EE.UU., Francia e Inglaterra, mientras que la Alemania Oriental estuvo orientada hacia la Unión Soviética. Durante ese periodo las dos Alemanias se rigieron por políticas diferentes hasta que en 1989 fue reunificada. Durante esos 44 años las dos partes desarrollaron diferentes modelos de psicólogos educativos. 
En la República Federal Alemania (Alemania Occidental), inmediatamente después de 1945, surgieron de manera inmediata clínicas de orientación infantil destinadas a ofrecer apoyo psicoterapéutico individual a los niños, muchos con secuelas psicológicas de la guerra. Sin embargo, estas clínicas no fueron del agrado de profesores y centros escolares. De esta manera, los psicólogos educativos iniciaron de nuevo su andadura en los años 50, instalándose centros específicos en las principales ciudades alemanas con el objeto de ofrecer apoyo psicológico e individualizado a aquellos alumnos que presentaban problemas de aprendizaje, a la vez que atendían a tareas de orientación dentro del centro escolar. El psicólogo formaba parte integral del sistema educativo y participaba con los profesores y directores de los centro en el desarrollo de actividades cruciales tales como la elaboración y diseño de materiales educativos. Un hito en el establecimiento del psicólogo educativo fue la resolución de Dortmund en 1966, en la que se trató de integrar y coordinar la amplia diversidad de actividades atribuibles al psicólogo y que figuraban, entre otras, la orientación, asistencia y apoyo continuo a los estudiantes de manera individual, incluyendo también apoyo a sus profesores y familiares más próximos, a promocionar y optimizar la enseñanza y la educación, la de incorporar diferentes puntos de vista de la psicología en la educación o la de coordinar actividades de diferentes profesionales e instituciones que estuviesen relacionadas con la educación del niño. Estas orientaciones fueron ampliamente aceptadas y formaron parte del quehacer diario del psicólogo educativo.

En la República Democrática Alemana la situación fue bastante diferente. Hasta el año 1973 el psicólogo educativo simplemente no existía. Fue en ese año cuando el Gobierno decidió que el psicólogo educativo debía formar parte del sistema educativo con la intención de que respondiese a las preguntas y problemas formulados por los profesores de infantil, primaria o secundaria, los educadores o los directores de los centros escolares públicos alemanes. Pero no se produjo un cambio sustancial hasta el año 1980, cuando se celebró el Congreso Internacional de Psicología en Leipzig. Hasta entonces, los líderes socialistas, económicos y educativos pensaban que la ideología socialista servía para todo. Este congreso puso de manifiesto las carencias de métodos y de formación en esta parte del país, lo que sirvió de acicate para incrementar el número de psicólogos educativos, a la vez que se fue aceptando al psicólogo dentro del sistema educativo. A pesar de la resistencia del sistema socialista hacia la práctica de una Psicología individual, estos nuevos psicólogos fueron capaces de desarrollar nuevas y variadas formas de asistir a maestros y profesores, estudiantes y familiares hacia la mejora del progreso educativo. Una de las pocas ventajas que tenía este modelo educativo era la existencia de una educación preescolar, que fue siempre parte integral del sistema, lo que facilitaba la transición del niño desde la guardería a la escuela reglada. Durante ese período se lograron desarrollar medidas de diagnóstico en la temprana infancia y programas de entrenamiento e intervención temprana siendo algunos de un nivel excelente (véase a este respecto Breuer y Weuffen, 1990).

Con la reunificación alemana en 1989 se produjo un intercambio frenético de información y de experiencias entre ambas maneras de entender la Psicología, llegando a ser la práctica del psicólogo educativo muy amplia y variada en sus actividades. Además, una de las características actuales positivas es la disposición de libertad que posee cada psicólogo para desarrollar aquellos aspectos del trabajo que considera más relevante. La otra cara de la moneda es que el psicólogo educativo puede sentirse muy sobrecargado por la gran cantidad de expectativas y obligaciones que genera. Otro factor de estrés a tener en cuenta es que el número de psicólogos educativos en Alemania es el más bajo de los países europeos por número de estudiantes (la ratio de un psicólogo educativo ha llegado a ser de 1 por cada 21.530 estudiantes, BDP 2008). Así se refleja en el reconocimiento que el Ministro de Educación alemán, Schleswig Holstein, pronunció en abril de 2010 con motivo de la inauguración de un congreso de Psicología. Decía así: 


\begin{abstract}
"El trabajo del psicólogo educativo es generalmente reconocido como un apoyo significativo del trabajo pedagógico en los centros escolares... Tengo un gran respeto por el trabajo de los psicólogos, que requieren de una empatía y unas habilidades de organización especiales. Trata el día a día con una amplia gama de tareas complejas y expectativas a las que el psicólogo debe enfrentarse. Esto merece un alto reconocimiento. Frente a los diversos desafíos de entre la densa red de recursos especiales que ofrece el futuro, la del psicólogo educativo es, sin duda, la más deseada" (Palabras de bienvenida del Dr. Ekkehard Klug, Ministro de Educación y Cultura de Schleswig Holstein/ Alemania, Abril 2010, en el Congreso titulado "Quo vadis Psicologia Educativa" organizada por los psicólogos educativos de Alemania en Rendsburg, que pertenece a la zona más baja de la prestación de Psicólogos en toda Europa (BDP 2008).
\end{abstract}

Tal y como se recoge en la Asociación de Psicología Educativa Alemana (The Sektion Schulpsychologie), el psicólogo educativo se define como un especialista que suele poseer también la formación de profesor (en algunos estados es un prerrequisito tener una oposición o acreditación de profesor de primaria o secundaria), y con una formación especializada en psicoterapia vocacional (http://www.schulpsychologie.de/sektion/index.htm). De esta manera, el psicólogo no sólo es considerado para tratar los problemas del día a día, sino que también participa en el diseño y desarrollo del centro en sí mismo. Tradicionalmente, el rol, funciones y responsabilidades del psicólogo educativo en Alemania tienen dos vertientes. Por un lado, proviene de una atención individualizada del estudiante, pero más que erigirse en defensor de ese alumno frente a los demás, es el experto que reúne información y opiniones de los padres, profesores y de otros profesionales sobre cómo cubrir mejor las necesidades de ese niño. Una segunda línea de trabajo se centra en el centro escolar en sí mismo, ofreciendo un servicio y apoyo que fortalezca el desarrollo educativo del centro. Usualmente, los psicólogos educativos disponen de sus gabinetes fuera de los centros escolares y desarrollan su actividad en varios centros. Por ello, no se consideran parte interna de la comunidad escolar o constituyente de un centro escolar particular, sino como un experto externo al centro al que asesora y asiste de manera regular.
Los psicólogos educativos disponen de sus propias asociaciones en cada uno de los dieciséis estados que configuran el país, aunque hay una de ámbito nacional, la asociación nacional de psicólogos educativos de Alemania (The Sektion Schulpsychologie im Berufsverband Deutscher Psychologinnen und Psychologen), dependiente de la Asociación Nacional de Psicólogos. En trece de los dieciséis estados que configuran Alemania se requiere de un diploma equivalente a un Máster en Psicología Educativa. En ciudades como Berlín o Hamburgo, además del máster, el psicólogo debe tener acreditación docente en centros públicos. A diferencia de Francia o Reino Unido, en Alemania no hay publicaciones o revistas especializadas o exclusivas en Psicología Educativa, sino revistas de psicología más multidisciplinar.

\section{La Psicología Educativa en Finlandia}

El establecimiento de la figura del psicólogo educativo en Finlandia, uno de los países cuyo sistema educativo es actualmente de los más desarrollados y eficientes, fue bastante rezagado respecto a otros países europeos. Este inicio comienza en 1938 cuando se crea en Helsinki el Centro de Orientación Infantil con el objeto de dar asistencia psicológica a las escuelas municipales de la capital (Laaksonen, Laitinen y Salmi, 2007). Pero no es hasta 1974 cuando la figura del psicólogo se desarrolla y consolida. Su función consistía, básicamente, en promocionar y mejorar el clima escolar, ofreciendo una atención individualizada y orientada hacia el tratamiento clínico. Esta concepción del psicólogo educativo fue modificándose paulatinamente como consecuencia de diversas reformas educativas. Una de ellas, la que se llevó a cabo en 2003, fue quizás la que más impacto causó en la función del psicólogo educativo, dirigiéndola hacia lo que se denomina "el bienestar del estudiante" (student welfire), y cuyo objetivo era buscar y promover aquellas condiciones que faciliten un proceso óptimo de aprendizaje en el estudiante, que conlleven un desarrollo físico y 
mental saludables, junto a un buen proceso de socialización (véase a este respecto, Laaksonen y cols., 2007).

El psicólogo educativo forma parte de un equipo multiprofesional denominado "equipo del bienestar del estudiante" (student welfire team) al que pertenecen, además, otros profesionales de la educación, como directores y profesores de los centros escolares, profesionales de la salud, profesores de educación especial y, en algunos casos, trabajadores sociales. Juntos coordinan la planificación de actividades de prevención e intervención en los distintos centros escolares, especialmente en los centros de educación primaria (de 6 a 12 años). Desde esta perspectiva, el psicólogo educativo trabaja en centros de educación municipal, social o de salud, siempre que estén orientados hacia la educación, ya sean estos públicos o instituciones privadas. Como tareas anexas se incluye la impartición de cursos de formación a otros profesionales de la educación (formación en psicoterapia, neuropsicología). Los casos más frecuentes que suelen atender se concentran en problemas sobre dificultades de aprendizaje y problemas familiares. En los niveles superiores también se hacen cargo del absentismo escolar y problemas de los estudiantes tratados individualmente.

Si bien la aplicación de pruebas y su evaluación forman parte de las herramientas básicas de las evaluaciones psicológicas, Finlandia tiene la particularidad de que estas evaluaciones están estrechamente ligadas al resto del equipo multiprofesional del centro. En este caso, las evaluaciones se planifican conjuntamente con los profesores del centro y sobre los resultados obtenidos se toman decisiones también conjuntas. Actualmente, esta faceta del psicólogo y la del equipo se ha incrementado con otros tipos de evaluaciones tanto médicas como neuropsicológicas en aquellos alumnos que presentan importantes dificultades de aprendizaje. Actualmente, existe un debate que gira en torno a la tendencia a concentrar el trabajo del psicólogo educativo hacia una atención más individualizada, como se viene haciendo hasta ahora o hacia el desarrollo de una intervención más sistémica, considerando al centro como un todo.
Esta tendencia se ha asociado con el desarrollo general del sistema educativo y de los centros escolares como organizaciones (Sipilä-Lähderkorpi, 2004).

Otra de las peculiaridades que presenta este país es que la formación del psicólogo educativo no parte de un programa específico de educación, sino que su formación universitaria se inicia con la preparación como psicólogo clínico y, una vez que obtiene la titulación, realiza prácticas bajo la supervisión de psicólogos experimentados. Hasta ahora la formación se inicia con el grado de psicología (3-4 años) y se completa con un máster de un mínimo de tres años. Los contenidos académicos son muy similares a los que se imparten en España y las funciones y responsabilidades radican fundamentalmente en la prevención, realizándose tan pronto como sea posible. Los principales cambios que se han producido actualmente han sido orientados hacia el incremento real de las necesidades educativas especiales. En este sentido, se ha desarrollado una regulación que acompaña a una reforma en la ley de educación básica potenciando las siguientes áreas: garantía de los servicios de bienestar; definir el papel y las funciones del equipo del bienestar del estudiante; confidencialidad; y definir el rol de las tareas y el estatus de los trabajadores dentro de este equipo multiprofesional.

\section{Situación actual del sicólogo educativo y de la Psicología Educativa en Europa}

Todo lo expresado hasta ahora en los apartados anteriores supone una pequeña muestra de la gran diversidad existente sobre la situación del psicólogo educativo entre los distintos países europeos. Tal diversidad afecta a todos los campos y situaciones, tales como la realización de los servicios psicológicos en el sistema educativo, la formación y especialización de los psicólogos, la prestación de una práctica supervisada, su representación profesional mediante asociaciones profesionales o sindicales (lo que afecta a las regulaciones salariales y condiciones laborales de los psicólogos), el estatus legal que 
los psicólogos poseen dentro del sistema educativo propio, la ratio de psicólogos por estudiante y profesores, la disponibilidad de instrumentos de evaluación adecuados, el desarrollo de oportunidades profesionales, o su formación continua. En las tablas que vienen a continuación se ilustran algunas de estas diferencias en los países que forman parte de la Unión Europea.

La Psicología aporta un elemento nuclear a la educación. Quizás por ello, algunos autores han establecido la analogía de la psicología es a la educación lo que la biología es a la medicina (Newcombe, 2000). El psicólogo educativo está reconocido internacionalmente por su papel esencial en el apoyo a la educación escolar. Todos los estados miembros europeos cuentan con los psicólogos en su sistema educativo. Así se recoge en los resultados de la OCDE PISA 2006 (véase tabla 1) donde se

Tabla1. Distribución de los psicólogos educativos por número, por alumnos escolarizados y por ratio psicólogo/alumno en cada uno de los países europeos y en otros países desarrollados (Fuente: Seifried, BDP 2008, Quelle: Jimerson, Stewart, Skokut, Cardenas, \& Malone. How Many School Psychologists are there in Each Country of the World? International Estimates of School Psychologists - and School Psychologist-to-Student Ratios.;http://education.ucsb.edu/jimerson/ NEW\%20IISP/ratios.html)

\begin{tabular}{lrcc}
\hline EUROPA & $\begin{array}{c}\text { Número de } \\
\text { psicólogos } \\
\text { educativos }\end{array}$ & $\begin{array}{c}\text { Número de } \\
\text { alumnos } \\
\text { escolarizados }\left(^{*}\right)\end{array}$ & $\begin{array}{c}\text { Ratio estimada } \\
\text { de niños } \\
\text { en edad escolar } \\
\text { por psicólogo }\end{array}$ \\
\hline Alemania & 982 & 9.311 .526 & $\mathbf{9 . 4 8 2}$ \\
Dinamarca & 880 & 815.963 & $\mathbf{9 2 7}$ \\
Escocia & 427 & 588.347 & $\mathbf{1 . 3 7 8}$ \\
Eslovaquia & 200 & 727.417 & $\mathbf{3 . 6 3 7}$ \\
Estonia & 150 & 160.763 & $\mathbf{1 . 0 7 2}$ \\
España & 3.600 & 4.657 .609 & $\mathbf{1 . 2 9 4}$ \\
Finlandia & 300 & 715.311 & $\mathbf{2 . 3 8 4}$ \\
Francia & 3.200 & 8.905 .092 & $\mathbf{2 . 7 8 3}$ \\
Grecia & 400 & 1.226 .554 & $\mathbf{3 . 0 6 6}$ \\
Holanda & 1.400 & 2.368 .410 & $\mathbf{1 . 6 9 2}$ \\
Hungria & 200 & 1.243 .642 & $\mathbf{6 . 2 1 8}$ \\
Irlanda & 128 & 678.142 & $\mathbf{5 . 2 9 8}$ \\
Lituania & 400 & 444.277 & $\mathbf{1 . 1 1 1}$ \\
Noruega & 945 & 711.305 & $\mathbf{7 5 3}$ \\
Reino Unido & 2.647 & 8.463 .502 & $\mathbf{3 . 1 9 7}$ \\
Rumania & 650 & 2.805 .682 & $\mathbf{4 . 3 1 6}$ \\
Suiza & 800 & 983.106 & $\mathbf{1 . 2 2 9}$ \\
OTROS PAISES & & & \\
Australia & 2.000 & 3.174 .492 & $\mathbf{1 . 5 8 7}$ \\
Canada & 3.500 & 4.672 .959 & $\mathbf{1 . 3 3 5}$ \\
Japón & 3.500 & 14.527 .200 & $\mathbf{4 . 1 5 1}$ \\
Estados Unidos & 32.300 & 48.649 .375 & $\mathbf{1 . 5 0 6}$ \\
\hline & & & \\
\hline
\end{tabular}

(*) Se ha utilizado el estándar de 12 años de educación obligatoria puede observar una distribución de los psicólogos educativos y ratio por alumno en los países europeos como en otros países desarrollados. Pero como puede observarse una vez más, existe una gran dispersión respecto a los datos recopilados.

La tabla 2 muestra algunos datos procedentes de los distintos países europeos relativos a cuestiones de legalidad profesional, identificados como "psicólogo educativo" (columna 1), de regulación o requerimiento de una licenciatura para ser psicólogo educativo o disponer de credencial para ejercer la profesión (columna 2), de la existencia de asociaciones profesionales (columna 3), de programas de formación universitaria específica para el psicólogo educativo (currículo específico para formar a estos profesionales, columna 4), y de la existencia de programas de doctorado específicos (columna 5). Como puede observarse en esta tabla también existen importantes divergencias entre los países que conforman la Unión Europea.

También resulta diferente el número promedio anual de publicaciones relacionadas con la Psicología Educativa en cada uno de los países (véase la tabla 3). Se observa, por ejemplo, una diferencia abismal entre Reino Unido sobre el resto de países europeos, tanto en la cantidad de documentos publicados como también en el impacto que producen sus publicaciones (H index 73). El caso de España es bien diferente, pues aunque el número de documentos publicados es importante, no tiene la misma repercusión internacional que otros países de su entorno ( $\mathrm{H}$ index 17).

Otro aspecto importante a tener en cuenta sobre la situación de la Psicología Educativa en Europa es conocer su peso en la disciplina y que resulta de poder compararla respecto a algún índice como el número de publicaciones respecto a otras especialidades de la psicología, como es la psicología clínica, social o aplicada. En la tabla 4 se recoge una muestra de la evolución del número de documentos publicados entre 1996 y 2009 y divididas por estas especialidades. La situación de la psicología educativa se sitúa en una zona intermedia, por delante de algunas áreas como la Psicología Aplicada o la Psi- 
Tabla 2. Distribución en los distintos países europeos de cuestiones relativas a la legalidad profesional identificados como "psicólogo educativo" (columna 1), sobre el requerimiento que para ser psicólogo educativo se requiera de una licenciatura, registrarse o disponer de credencial (columna 2), existencia de asociaciones profesionales de Psicología Educativa (columna 3), existencia de programas de formación universitaria específica para el psicólogo educativo (columna 4) y sobre la existencia de programas de doctorado específicos para el psicólogo educativo (columna 5). (Fuente: Seifried, BDP 2008)

\begin{tabular}{|c|c|c|c|c|c|}
\hline País & $\begin{array}{c}1 \\
\text { Profesionales identificados } \\
\text { y empleados con las } \\
\text { características de } \\
\text { "psicólogo educativo" }\end{array}$ & $\begin{array}{c}\mathbf{2} \\
\text { Regulaciones (o leyes) } \\
\text { que requieren del } \\
\text { "psicólogo educativo" } \\
\text { a obtener una } \\
\text { licenciatura, registrarse } \\
\text { o disponer de credencial }\end{array}$ & $\begin{array}{c}\mathbf{3} \\
\text { Asociaciones } \\
\text { profesionales del } \\
\text { "psicólogo educativo" } \\
\text { (incluyendo la sección } \\
\text { de psicología educativa } \\
\text { dentro de la asociación } \\
\text { profesional nacional) }\end{array}$ & $\begin{array}{c}\mathbf{4} \\
\text { Programas } \\
\text { universitarios dirigidos } \\
\text { a la formación del } \\
\text { "psicólogo educativo" } \\
\text { (currículo específico } \\
\text { para formar a estos } \\
\text { profesionales) }\end{array}$ & $\begin{array}{c}\mathbf{5} \\
\text { Programas de } \\
\text { doctorado específicos } \\
\text { para el psicólogo } \\
\text { educativo }\end{array}$ \\
\hline
\end{tabular}

\begin{tabular}{|c|c|c|c|c|c|}
\hline Alemania & $\mathbf{S i}$ & $\mathbf{S i}$ & $\mathbf{S i}$ & $\mathbf{S i}$ & No \\
\hline Austria & $\mathbf{S i}$ & $\mathbf{S i}$ & No & No & No \\
\hline Bélgica & $\mathbf{S i}$ & No & $\mathbf{S i}$ & $\mathbf{S i}$ & No \\
\hline Chipre & $\mathbf{S i}$ & $\mathbf{S i}$ & $\mathbf{S i}$ & $\mathbf{S i}$ & $\mathbf{S i}$ \\
\hline Dinamarca & $\mathbf{S i}$ & $\mathbf{S i}$ & $\mathbf{S i}$ & $\mathrm{Si}$ & No \\
\hline Eslovaquia & $\mathbf{S i}$ & $\mathrm{Si}$ & $\mathbf{S i}$ & No & $\mathrm{Si}$ \\
\hline Eslovenia & $\mathbf{S i}$ & No & $\mathbf{S i}$ & No & No \\
\hline España & $\mathbf{S i}$ & $\mathbf{S i}$ & No & $\mathbf{S i}$ & No \\
\hline Finlandia & $\mathrm{Si}$ & $\mathrm{Si}$ & $\mathbf{S i}$ & No & No \\
\hline Francia & $\mathbf{S i}$ & $\mathbf{S i}$ & $\mathbf{S i}$ & $\mathbf{S i}$ & No \\
\hline Grecia & $\mathrm{Si}$ & $\mathrm{Si}$ & $\mathbf{S i}$ & $\mathrm{Si}$ & $\mathrm{Si}$ \\
\hline Holanda & $\mathbf{S i}$ & No & $\mathbf{S i}$ & No & No \\
\hline Hungría & $\mathbf{S i}$ & $\mathrm{Si}$ & $\mathbf{S i}$ & $\mathbf{S i}$ & No \\
\hline Irlanda & $\mathbf{S i}$ & No & $\mathbf{S i}$ & $\mathbf{S i}$ & No \\
\hline Italia & $\mathbf{S i}$ & No & $\mathbf{S i}$ & $\mathrm{Si}$ & No \\
\hline Noruega & $\mathrm{Si}$ & No & $\mathrm{Si}$ & No & No \\
\hline Polonia & No & No & No & No & No \\
\hline Portugal & $\mathbf{S i}$ & No & No & $\mathrm{Si}$ & No \\
\hline R.U.: Escocia & $\mathrm{Si}$ & $\mathbf{S i}$ & $\mathbf{S i}$ & $\mathbf{S i}$ & $\mathbf{S i}$ \\
\hline R.U.: Inglaterra y Gales & $\mathrm{Si}$ & $\mathrm{Si}$ & $\mathrm{Si}$ & $\mathbf{S i}$ & $\mathbf{S i}$ \\
\hline Republica Checa & $\mathbf{S i}$ & No & $\mathbf{S i}$ & $\mathbf{S i}$ & No \\
\hline Rumania & $\mathbf{S i}$ & $\mathbf{S i}$ & $\mathbf{S i}$ & $\mathbf{S i}$ & $\mathbf{S i}$ \\
\hline Rusia & $\mathbf{S i}$ & No & $\mathbf{S i}$ & $\mathbf{S i}$ & No \\
\hline Suecia & $\mathrm{Si}$ & $\mathrm{Si}$ & $\mathrm{Si}$ & $\mathrm{Si}$ & No \\
\hline Suiza & $\mathrm{Si}$ & No & $\mathbf{S i}$ & $\mathrm{Si}$ & No \\
\hline
\end{tabular}

Tabla 3. Promedio del número de documentos publicados anualmente y relacionados con la psicología educativa en cada uno de los países de la Europa Occidental durante el periodo comprendido entre 19962009 (Fuente: Scimago Lab, Copyright 2007-2011. Data Source: Scopus@)

\begin{tabular}{|c|c|c|c|c|c|c|c|}
\hline & País & Documentos & $\begin{array}{l}\text { Documentos } \\
\text { citados }\end{array}$ & Citas & Auto-citas & $\begin{array}{c}\text { Citas por } \\
\text { documento }\end{array}$ & Hindex \\
\hline 1 & Reino Unido & 2.160 & 2.052 & 30.506 & 9.231 & 17,39 & 73 \\
\hline 2 & Holanda & 745 & 730 & 10.348 & 2.755 & 16,97 & 42 \\
\hline 3 & Alemania & 660 & 639 & 10.565 & 2.837 & 24,15 & 49 \\
\hline 4 & España & 399 & 388 & 1.665 & 577 & 7,50 & 17 \\
\hline 5 & Francia & 376 & 366 & 3.275 & 900 & 12,11 & 29 \\
\hline 6 & Suecia & 279 & 278 & 4.107 & 1.064 & 19,07 & 31 \\
\hline 7 & Italia & 217 & 209 & 2.600 & 387 & 18,06 & 25 \\
\hline 8 & Bélgica & 210 & 207 & 2.661 & 502 & 19,18 & 28 \\
\hline 9 & Finlandia & 188 & 186 & 2.577 & 567 & 17,66 & 27 \\
\hline 10 & Noruega & 147 & 145 & 1.669 & 198 & 15,86 & 21 \\
\hline 11 & Turquía & 138 & 136 & 682 & 165 & 7,00 & 12 \\
\hline 12 & Suiza & 127 & 126 & 1.258 & 202 & 14,24 & 18 \\
\hline 13 & Irlanda & 79 & 73 & 482 & 83 & 7,51 & 10 \\
\hline 14 & Grecia & 75 & 70 & 700 & 100 & 13,57 & 14 \\
\hline 15 & Portugal & 49 & 49 & 383 & 35 & 13,28 & 11 \\
\hline 16 & Austria & 46 & 42 & 754 & 91 & 22,67 & 16 \\
\hline 17 & Dinamarca & 42 & 39 & 563 & 94 & 26,48 & 12 \\
\hline 18 & Chipre & 28 & 28 & 173 & 25 & 8,01 & 8 \\
\hline 19 & Islandia & 14 & 12 & 191 & 8 & 17,94 & 6 \\
\hline
\end{tabular}


Tabla 4. Evolución del número de documentos publicados en Psicología y divididos por categorías en países de Europa Occidental durante los años 1996-2009. (Fuente: Scimago Lab, Copyright 2007-2011. Data Source: Scopus®)

\begin{tabular}{|c|c|c|c|c|c|c|c|c|c|c|c|c|c|c|}
\hline Periodo en años & 1996 & 1997 & 1998 & 1999 & 2000 & 2001 & 2002 & 2003 & 2004 & 2005 & 2006 & 2007 & 2008 & 2009 \\
\hline Psicología Aplicada & 139 & 122 & 175 & 169 & 199 & 216 & 218 & 241 & 252 & 315 & 309 & 428 & 459 & 631 \\
\hline Psicología Clínica & 215 & 235 & 206 & 214 & 259 & 269 & 276 & 431 & 502 & 639 & 699 & 788 & 937 & 1.109 \\
\hline $\begin{array}{l}\text { Psicología Educativa y } \\
\text { desarrollo } \\
\text { Psicología Experimental }\end{array}$ & 192 & 193 & 208 & 222 & 221 & 250 & 389 & 340 & 396 & 393 & 459 & 626 & 657 & \\
\hline $\begin{array}{l}\text { Cognitiva } \\
\text { Psicología Fisiológica y }\end{array}$ & 532 & 572 & 449 & 498 & 522 & 608 & 624 & 678 & 745 & 797 & 963 & 1.078 & 1.259 & 1.361 \\
\hline Neuropsicología & 662 & 683 & 648 & 764 & 965 & 886 & 949 & 883 & 919 & 1.011 & 833 & 802 & 904 & 1.000 \\
\hline Psicología (conjunto) & 2.640 & 2.698 & 2.673 & 3.194 & 3.278 & 3.181 & 3.574 & 4.006 & 3.818 & 4.148 & 4.352 & 4.789 & 4.896 & 5.127 \\
\hline Psicología Social & 190 & 185 & 195 & 145 & 175 & 229 & 226 & 263 & 289 & 345 & 364 & 474 & 609 & 832 \\
\hline
\end{tabular}

cología Social y por detrás de otras como la Psicología Clínica o la Experimental.

\section{Necesidad de una integración en la formación y profesionalización común del psicólogo educati- vo en los países de la Unión Europea}

Todas estas divergencias descritas en los apartados anteriores sobre la situación del psicólogo educativo en diversos países europeos nos llevan a plantear la necesidad de consenso, una obligada búsqueda por encontrar puntos de encuentro que permitan generar una integración del estatus del psicólogo educativo en toda la Comunidad Europea. Generar, en definitiva, un marco de referencia europeo. Afortunadamente ya se han puesto en marcha una serie de iniciativas europeas que describiremos en los apartados siguientes.

\section{A) Sobre el fomento de una Federación Europea de Asociaciones de Psicólogos (European Federation of Psychologists' Associations, EFPA) y el certificado europeo EuroPsy.}

Desde la pasada década ya se viene produciendo una intensa actividad en Europa sobre la necesidad de integrar las funciones y formación especializada del psicólogo educativo en toda Europa. En esta dirección apuntan las propuestas realizadas por la Federación Europea de Asociaciones de Psicólogos (European Federation of Psychologists' Associations, E.F.P.A. http://www.efpa.eu), que ha venido desarrollado un marco de referencia europeo para la educación y la formación del psicólogo educativo, el "EuroPsy", El EuroPsy es un Certificado Europeo de Psicología Educativa y cuyo objeto no es otro que el que sirva en el futuro como una tarjeta profesional reconocida en toda Europa. El certificado se corresponde con la necesidad de que las políticas europeas coincidan en la mejora de la movilidad y en la creación de una calidad de formación común que sea estándar de la profesión. Con este motivo se desarrolló en 2007 la Red Europea de Psicólogos en el Sistema Educativo (Network of European Psychologists in the Educational System NEPES, http://www.nepes.eu), concebida bajo el paraguas de la EFPA con el fin de mejorar la cooperación europea en este grupo de profesionales.

Aunque se trata de un desarrollo aún reciente, esta red europea está siendo reforzada por proyectos europeos financiados como el proyecto ESPIL (European School Psychologists Improve Lifelong Learning) que ha permitido reunir a representantes de la Psicología nacional de los distintos países europeos y a las asociaciones de Psicología Educativa en su conjunto para: a) informar sobre las políticas europeas pertinentes; b) comparar la situación de los psicólogos educativos de toda Europa; c) analizar la influencia de las políticas europeas en materia de educación y formación en la profesión; y d) desarrollar una perspectiva sobre cómo los psicólogos pueden contribuir a las políticas europeas relacionadas con en el aprendizaje continuo (European Lifelong Learning Policies, LLP). 
B) Sobre la identidad del psicólogo educativo, sus funciones y áreas de intervención en el ámbito europeo

Entre los temas que han sido objeto de debate en la EFPA sobresale el referente de nombres y etiquetas bajo las que se designan a un psicólogo educativo en los distintos países europeos e, incluso, dentro del mismo país y la necesidad de unificar la identidad del psicólogo bajo un mismo término. Así, por ejemplo, un psicólogo educativo se identifica como "psicólogo de la escuela o psicólogo escolar", "orientador", "psicopedagogo", "asesor psicopedagógico", "consultor educativo". La definición más consensuada del psicólogo educativo en Europa es la de aquel profesional que posee una licenciatura en psicología y experiencia o formación específica en el campo o grado en Psicología de la Educación (EFPA, 2001, http:// www.efpa.eu). Este consenso, redactado en un informe de la EFPA afecta, por tanto, a todos los psicólogos que trabajan en el ámbito educativo, ya trabajen en centros públicos o privados de la escuela infantil, escuela primaria o centros de enseñanza secundaria, cubriendo así un rango aproximado de alumnos cuyas edades se sitúan entre los 2 y los 20 años. A estos profesionales también se agregan aquellos psicólogos que trabajan en servicios sociales y aprendizaje no formal como los responsables de los servicios psicopedagógicos municipales, servicios de orientación u otros centros o instituciones en los que los psicólogos realizan funciones educativas. Estos psicólogos se conocerán en términos globales como "psicólogo educativo" (aunque las siglas en inglés aparecen indistintamente como School Psychologist o Educational Psychologist). Se prefiere, en cualquier caso el término de psicología educativa y psicólogo educativo (Educational Psychology, Educational Psychologist).

Actualmente, la Psicología Educativa constituye una de las ramas más importantes de la psicología aplicada. Requiere de un bagaje de conocimientos complejos entre los que se incluye un buen dominio de la Psicología Evolutiva y desarrollo humano, de Psicopatología Infantil y Juvenil, de la psi- cología de las organizaciones, el impacto de las ciencias sociales, de la neurociencia, del papel de la cultura, de la teoría del aprendizaje y adquisición de conocimientos, del impacto del funcionamiento de los padres y la familia, de los principios de la enseñanza eficaz y las escuelas efectivas en entornos educativos. Dentro de la EFPA se ha establecido una matriz que muestra una amplia gama de actividades profesionales distribuidas en tres áreas: prevención, evaluación e intervención, que se especifican en relación con el individuo, grupo, sistema y nivel de la sociedad, como puede observarse en la tabla 1.

C) Sobre la necesidad de realizar análisis rigurosos sobre la situación del sistema educativo y sus posibles mejoras en Europa

Aunque se reconoce que los psicólogos educativos desempeñan un papel muy significativo en la educación escolar, esta afirmación contrasta, sin embargo, con la ausencia de datos que permitan analizar los recursos psicológicos de los países de la UE o un análisis de los costes y beneficios de los resultados educativos que pudiesen derivarse de los servicios psicológicos. De esta manera, los responsables políticos no disponen en la actualidad de evidencia alguna sobre si los recursos disponibles resultan o no eficientes, de si se corresponden con otros estándares de calidad desarrollados en otros países o de si estos recursos pueden mejorarse para alcanzar los objetivos deseados. Aún es más, los datos de la OCDE -según reflejan los estudios PISA sobre el rendimiento de los sistemas educativos de su entorno- concluyen que la mayoría de los países no disponen de datos sobre los psicólogos educativos (http://stats.oecd.org/Index.aspx?DatasetCode= RPERS).

Si asumimos que la educación es un factor clave en el desarrollo del capital humano como también lo es para la recuperación de la crisis económica, nuestros gobernantes deberían prestan más atención a los resultados de la educación y de la formación propia de cada país, ya se base ésta en análisis cuantitativos 
Tabla 5. Perfiles profesional del psicólogo educativo visto como un proceso dinámico

\begin{tabular}{|c|c|c|c|c|}
\hline FUNCION/NIVEL & SOCIEDAD & SISTEMA EDUCATIVO & GRUPO & INDIVIDUO \\
\hline PREVENCION & $\begin{array}{l}\text { Proporcionar información } \\
\text { sobre condiciones de vida, } \\
\text { desarrollo psicológico, } \\
\text { factores de riesgo y pro- } \\
\text { tección. } \\
\text { Iniciar programas de } \\
\text { investigación en áreas } \\
\text { relevantes, promover las } \\
\text { reformas necesarias sobre } \\
\text { la influencia y las normas } \\
\text { legales en todas las áreas } \\
\text { relativas a entornos de } \\
\text { aprendizaje óptimo. }\end{array}$ & $\begin{array}{l}\text { Proporcionar asesoramien- } \\
\text { to a la administración, a } \\
\text { los directivos, a maestros } \\
\text { y padres, a alumnos y a } \\
\text { sus representantes. } \\
\text { Iniciar y coordinar proyec- } \\
\text { tos en las áreas pertinen- } \\
\text { tes. Promover cambios } \\
\text { estructurales puedan resul- } \\
\text { tar útiles; } \\
\text { Promover cambios en el } \\
\text { pensamiento pedagógico } \\
\text { cuando sea necesario, dise- } \\
\text { ñando planes de acción y } \\
\text { posibilitar una evaluación } \\
\text { sobre lo aplicado. }\end{array}$ & $\begin{array}{l}\text { Proporcionar asesoramien- } \\
\text { to y supervisión; Estimular } \\
\text { el trabajo en equipo. } \\
\text { Observar el comporta- } \\
\text { miento del grupo y su } \\
\text { interacción; } \\
\text { Proporcionar información } \\
\text { y conocimiento, apoyo a la } \\
\text { aplicación y evaluación de } \\
\text { proyectos relevantes (e.g., } \\
\text { el aprendizaje, la intimida- } \\
\text { ción, a la prevención de } \\
\text { drogas, a la salud mental, } \\
\text { a la gestión de crisis). }\end{array}$ & $\begin{array}{l}\text { Ofertar consultas de orien- } \\
\text { tación y supervisión; } \\
\text { Observar y evaluar los sín- } \\
\text { tomas individuales y habi- } \\
\text { lidades de interacción. }\end{array}$ \\
\hline
\end{tabular}

\section{EVALUACION}

\begin{abstract}
Analizar los datos de muestras para la documentación sobre el grupo, centro, regional o nacional;

Definir las medidas psicológicas, los conocimientos, las habilidades y métodos en la evaluación.
\end{abstract}

Ofrecer seguimiento a los proyectos y los métodos aplicados, a los planes de acción, al conocimiento de transferencia a situaciones nuevas o aplicadas.
Entrevistas, cuestionarios, grabaciones en vídeo, etc., para identificar y examinar los grupos con el fin de distinguir aquellos aspectos culturales y normas sociales y su interacción, nivel intelectual y las necesidades, la motivación para el cambio, etc.
Evaluar a través de entrevistas, cuestionarios u otro tipo de pruebas el funcionamiento intelectual, las capacidades de aprendizaje, de conducta, las alteraciones emocionales o de personalidad, problemas sociales o familiares, así como determinar la necesidad de un examen más detallado (i.e., un examen neurológico u clínico). La evaluación debe estar orientada hacia el tratamiento y hacia la inclusión del alumno en el sistema educativo.

\section{INTERVENCION}
Influir en aquellas refor- mas necesarias relativas a entornos de aprendizaje óptimo; Facilitar el acceso a los servicios psicológi- cos y estructuras de apoyo escolar o a aquellas com- petencias necesarias para la práctica psicológica; Mejorar la calidad del psi- cólogo educativo en todos los niveles (escolar, local, regional, comunitaria o nacional).
Iniciar proyectos para grupos de directores de centro/ de docentes/ de alumnos / padres, para la supervisión de la formación, de grupos terapéuticos (i.e., la terapia familiar).

Desarrollar nuevos métodos y materiales para el uso psicológico y docente.
Facilitar cuando sea indispensable la educación especial, la formación específica, la terapia, el cambio de centro, el cambio de clase, el desarrollo de nuevos materiales (pruebas y formación), de asistencia externa a la exploración.

Participar en los informes suplementarios (postgrado) de formación. o cualitativos, así como en las comparaciones internacionales que se realizan regularmente (como ocurre con el informe PISA) y en la eficacia obtenida como consecuencia de invertir en educación y formación. La calidad de la educación y la formación se convierte así en una estrategia esencial para los gobiernos de cada país y, conjuntamente, los de la
Unión Europea. Puesto que se trata de una estrategia tan importante, las decisiones políticas sobre reformas educativas sólo se deberían llevar a cabo sobre la base de datos objetivos, válidos y fiables, como los que aporta la Red Europea sobre de Economía Educativa (European Network on Economics of Education, EENEE). 


\section{D) ¿Cómo afecta la crisis actual al desarrollo de la Psicología Educativa en la UE? La Psico- logía Educativa en términos económicos}

Debido a la crisis económica actual y al igual que ocurre en otros campos, la administración pública de toda Europa ha recortado sus presupuestos en educación. Esta situación también afecta a la contratación de psicólogos educativos, también sometidos a estas leyes del mercado, "desconectándose" su contratación en tiempos de crisis o "activándose" en tiempos de bonanza. Algunas encuestas (como la realizada por la Federación Sindical de Trabajadores de la Educación en 2009) revelan una reducción en los presupuestos de educación entre el 5 al 10\% en la mayor parte de los países europeos, que afecta también a la contratación laboral. En Francia, por ejemplo, se reemplaza sólo uno de cada dos psicólogos educativos que se jubilan. Estos recortes presupuestarios conllevarán, tal y como predice el informe emitido por el Consejo de la UE en 2010 sobre la protección social e inclusión, que las desigualdades entre los jóvenes aumentará inevitablemente al limitar su acceso a los servicios de apoyo educativo y de salud, creará mayores costes sociales a largo plazo afectando, especialmente, a los grupos más vulnerables (trabajadores poco cualificados, discapacitados, personas socialmente desfavorecidas, minorías inmigrantes).

Otra manera en que puede evaluarse estos recortes presupuestarios es en el tipo de actividades que el psicólogo educativo realiza en tiempos de crisis y que está determinado por la ratio psicólogo/alumnos. La ratio psicólogo educativo/estudiante tiene un impacto directo en el tipo de servicio proporcionado. Así, cuando la proporción de un psicólogo educativo es menor a 1.000 estudiantes, éste dedica mayor tiempo a actividades relacionadas con la intervención y prevención. Pero cuando la ratio supera los 1000 estudiantes, su tiempo se concentra más en actividades de evaluación. Si no hay recursos disponibles para ampliar el ámbito de aplicación del psicólogo educativo, los datos sugieren que tanto la dirección del centro como otros profesionales de la educación prefieren que el psicólogo se concentre entonces en un trabajo más individualizado. Esto lleva a la conclusión de que una disminución de los puestos de trabajo se traduce en más tiempo que el psicólogo invierte en actividades menos eficientes de evaluación, llegando sólo a unos pocos alumnos. Por el contrario, se pierde en la prevención primaria, más rentable para un gran número de estudiantes y para la comunidad escolar en general. Un ejemplo que ilustra la ratio de costes-beneficios de los psicólogos educativos se encuentra en el informe realizado por Seifried (2008) en Berlín, Alemania. El extracto que hemos seleccionado dice así:

De acuerdo con los resultados del estudio internacional, el porcentaje relativo de las poblaciones estables estudiante que necesitan atención psicológica se estima en alrededor del $10 \%$. Un psicólogo educativo atiende en Berlín a unos 6.200 estudiantes. Tan sólo un 2,6\% de toda esta población estudiantil de Berlín contó con el apoyo adecuado debido a la falta de recursos de psicólogos educativos. Desde 2003, Berlín se ha reducido el número de recursos en un $40 \%$.

Del 100\% del tiempo que el psicólogo educativo dedica a problemas de aprendizaje lo distribuye de la siguiente manera: el $30 \%$ de su tiempo lo dedica a problemas y dificultades de la lectura y escritura, otro $30 \%$ lo emplea en tareas de asesoramiento a profesores, un $10 \%$ se centra en necesidades educativas especiales, el $10 \%$ de su tiempo ofrece apoyo psicológico a alumnos repetidores, el $3 \%$ a la dislexia, $3 \%$ a discalculia, y entre $2-4 \%$ a temas relacionados con los superdotados.

Los costes por alumno en situación de riesgo se estiman en los siguientes datos: Costes por cada alumno repetidor asciende a coste anual de 5.000 por alumno. Por un apoyo privado que ofrezca ayudas de aprendizaje suplementarias asciende al año 4.000 por alumno; si la ayuda que requie re es una psicoterapia privada el coste se eleva entre 5.000 - 7.500 por niño y año. Si necesita de atención psiquiátrica en un hospital para niños el coste se dispara a 72.000 por niño y año, la misma cantidad que se requiere para recluir a un niño o un adolescente en un centro de menores.

El 10\% de los estudiantes en Berlín (aproximadamente 35.000 estudiantes) repiten clase. Como se señalaba en el párrafo anterior, por cada alumno repetidor se incrementa su coste anual en 5.000 . Si cada uno de estos estudiantes pudiese ser integrado en la escuela con el apoyo de un psicólogo educativo reduciría significativamente los costes en la educación, en la salud y lo de carácter social. Con que un psicólogo educativo evitara tan sólo que 10 alumnos repitiesen, el coste ahorrado superaría sus honorarios anuales. 


\section{A modo de conclusión: ¿Cómo desarrollar un marco de referencia europeo que establezca normas de calidad en la formación continua y cualificación del psicólogo educativo?}

Los resultados del Cuestionario ESPIL 2010 indican grandes diferencias en toda Europa que hacen muy compleja la elaboración de un marco de referencia europeo para el psicólogo educativo. Algunas de estas diferencias ya las hemos expuesto en los anteriores apartados pero hay muchas otras. Una de ellas es la ratio de cada psicólogo por profesor y estudiante. Por ejemplo, mientras que un psicólogo educativo español actualmente atiende a una comunidad de cerca de 79 profesores, un búlgaro se ocupa de 300 maestros; mientras que en Dinamarca un psicólogo se responsabiliza de una población de 800 estudiantes de promedio, un alemán lo hace sobre 12.400 alumnos. Estas diferencias también se establecen en relación a apoyo de otros profesionales de la educación. Por ejemplo, considerando que un psicólogo educativo de Lituania no dispone de apoyo adicional de trabajadores sociales, un psicólogo noruego puede contar con la colaboración de hasta 26 trabajadores sociales. En los sistemas educativos de Noruega, Austria, Finlandia y Suecia, el psicólogo educativo tiene la posibilidad de cooperar con un mayor número de otros profesionales (profesor de educación especial, médicos escolares, enfermeras escolares, consejeros educadores, especialistas como logopedas, fisioterapeutas, trabajadores sociales, etc.), en comparación con otros países de su entorno que no disponen de tales medios humanos. Este hecho que supone un valioso apoyo para los centros escolares, puede significar también una mayor competencia y solapamiento de funciones y responsabilidades. Otro apartado son los salarios comparables para las profesiones de cualificación similar. Los resultados del Cuestionario ESPIL 2010 también indican que la mayoría de los países europeos ofrecen un salario similar al de los profesores de enseñanza secundaria. Sin embargo, el salario medio anual de un psicólogo en Europa varía entre 6.000 y 74.000 , lo que no es exclusivamente atri - buible a las diferencias referidas a la carestía de la vida de cada país.

Existen, además, otros problemas relacionados con los instrumentos de evaluación que utilizan los psicólogos educativos y que se muestran obsoletos ante los nuevos retos educativos. Así lo revela al menos un estudio realizado por Haney y Evans (1999) en el que se investigaba el uso de métodos de evaluación menos tradicionales como la evaluación funcional o dinámica. Los resultados indicaron que si bien la población europea está cambiando progresivamente hacia una diversidad cultural y plurilingüe, lo que demanda un tipo de evaluación más funcional, lo cierto es que los instrumentos siguen dependiendo en gran medida de las técnicas tradicionales de evaluación (La escala de Wechsler para niños, la escala de inteligencia de Stanford-Binet, etc.). Esto supone una dificultad añadida para el psicólogo, pues las herramientas de evaluación disponibles no coinciden con las necesidades actuales de aquellos sistemas educativos que contemplan aulas multilingües y multiculturales y políticas de educación inclusiva, y donde se necesitan nuevas herramientas que aporten sugerencias para las medidas de apoyo futuras. Actualmente no hay ninguna posibilidad para evaluar, pongamos por caso, la dislexia en un niño de primaria en Luxemburgo, hijo de padre español y madre sueca, y que ha sido inscrito en un centro infantil de habla francesa. No hay herramientas que cubran las complejas circunstancias culturales para la ortografía y las dificultades de lectura. Todo esto urge el desarrollo de nuevas herramientas de evaluación, lo que se está convirtiendo en objeto de investigación reciente (véase Germeijs, Verschueren y Van der Vliet, 2003).

Con el Certificado Europeo de Psicología, Europsy se abre un punto de referencia común de estándares mínimos de calidad en la educación y la formación de psicólogos europeos. Entre otras cosas, este certificado propone un periodo de formación en Psicología de cinco años más otro de práctica supervisada. También contempla la posibilidad de una actualización profesional continua. EuroPsy reúne buena parte de las peticiones de las políticas 
europeas sobre el reconocimiento, transparencia y comparabilidad de cualificaciones profesionales y movilidad de trabajo. Este punto de referencia todavía no se ha aplicado a la especialización de la Psicología Educativa dada la enorme dispersión existente entre los distintos países europeos, como ya hemos analizado. Hasta el momento sólo un país, Suecia, parece cumplir con todos los requisitos que EuroPsy demanda. Hay, sin embargo, otras interesantes propuestas que están siendo estudiadas. Holanda, por ejemplo, propone un periodo de dos años de educación postmaster para los psicólogos educativos. Austria y Luxemburgo son partidarios de introducir varios años de prácticas bajo supervisión que incluya, una vez finalizada, exámenes especiales para obtener la acreditación. España, por su parte, insiste en la introducción de un máster europeo sobre Psicología Educativa.

Hay en marcha otras propuestas como la creación de un grupo de estudio europeo que investigue el desarrollo de nuevos instrumentos de evaluación, así como de establecer unos estándares de calidad relacionada con las condiciones de trabajo adecuadas. Hoy se acepta internacionalmente un mínimo de un psicólogo por cada 1000 estudiantes. Igualmente y debido a la naturaleza siempre cambiante de la sociedad y del sistema educativo, es de vital importancia una actualización de conocimientos para mantenerse al día con nuevos conocimientos, nuevos métodos y herramientas, con el desarrollo de la investigación científica y la teoría, especialmente las neurociencias y el acceso a nuevos métodos experimentalmente probados (por ejemplo, pruebas, tratamientos, consultas, etc.) con el fin de asegurar el rol profesional. Sobre este respecto y en comparación con los docentes, los psicólogos representan un pequeño grupo profesional dentro del sistema educativo y su actualización resulta relativamente costosa a menos que se beneficie de la cooperación europea. El modelo de cursos de formación podría organizarse desde un Organismo Internacional de Psicología Educativa que promocionase la calidad profesional y el intercambio de experiencias de colegas de distintos países. En un centro como éste podría desplazarse, pongamos por caso, un psicólogo francés que participan en cursos de formación en francés organizado en Luxemburgo o un alemán que participa en cursos de formación en España. Situaciones como éstas permitiría una oferta enriquecida de cursos de formación, mejoraría la movilidad profesional y la cooperación, así como la competencia cultural y el conocimiento de otras lenguas. Otra vía posible posibilidad es el establecimiento de un Centro Europeo de Formación Profesional para el psicólogo educativo (European Vocational Training Centre) y que podría ser también una solución económicamente viable y una contribución positiva para mejorar la calidad en toda Europa. La tarea de este Centro Europeo de Formación sería la de coordinar la capacitación nacional hacia un nivel europeo estándar (por ejemplo, calendario europeo de las ofertas de capacitación, coordinar el desarrollo de estándares de calidad comparables, e incluso ofertas de empleo).

En resumen, se puede afirmar que la aplicación del Marco Europeo de Cualificaciones para el aprendizaje continuo basado en los resultados del aprendizaje y el conocimiento formulado, habilidades y competencias sólo ha comenzado a ser aplicada a la educación y la formación del psicólogo educativo en toda Europa. Todavía resta restablecer algunas lagunas y necesidades para poder completar este marco europeo donde se pueda establecer descriptores comparables sobre competencias básicas de los profesionales y que coincidan con los planes de estudios basados en competencias. Igualmente, se requiere de guías de orientación y apoyo durante la fase de formación de los psicólogos educativos, desarrollar estándares de calidad comparables incluyendo la cualificación de formadores e incentivos para el aprendizaje permanente. La cooperación europea también necesita nutrirse de planes de desarrollo de aprendizaje entre iguales, del intercambio de experiencias, de coordinación de proyectos europeos, todo ello promovidos desde las universidades y de las asociaciones profesionales europeas. También se requiere establecer modelos comparables de planes de estudio basados en competencias, teniendo en 
cuenta las necesidades profesionales, normas comparables de calidad de la educación especializada, incluidas la supervisión como parte integrante del trabajo profesional, especialmente durante la fase de prácticas. Todo ello nos supone un reto y esfuerzo considerables que puede valer la pena de cara al futuro del psicólogo educativo.

\section{Referencias}

Binet, A., y Vaschide, N. (1897). La psychologie à l'ecole primaire. LÁnnée Psychologique, 4,1-14.

Breuer, H. y Weuffen, M. (1990). Gut vorbereitet auf das Lesen-und Schreibenlernen? Berlin: Deutscher Verlag der Wissenschaften.

Bristish Psychological Society (2005 a, February 28). Educational psychology statement [Position statement]. Leicester: Author.

Dunkel, L. (2007). School Psychology in Germany. In S. R. Jimerson, T. D. Oakland \& P. T. Farrell (Eds.), The Hanbook of International School Psychology (pp.123-134). London, U.K.: Sage Publications, Inc.

Foucault, M. (1923). Observations et experiences de psychologie scolaire. Paris: Presses Universitaires de France.

Germeijs, V., Verschueren, K. y Van der Vliet, L. (2003). Een rondvraag naar diagnostische middelen en behoeften in de CLB's. Caleidoscoop, 1,2, 22-26.

Guillemard, J. C. (2007). School Psychology in France. In S. R. Jimerson, T. D. Oakland \& P. T. Farrell (Eds.). The Hanbook of International School Psychology (pp.113-122). London, U.K.: Sage Publications, Inc.

Guillemard, J. C. y Guillard, S. (2007). Manuel practique de psychologie en milieu educative. Paris: Mason.

Haney, M. R. y Evans, G. (1999). National survey of school psychologists regarding the use of dynamic assessment and other non traditional techniques. Psychology in the Schools, 36, 4, 295-304.

Laaksonen, P., Laitinen, K., y Salmi, M. (2007). School Psychology in Finland. In S. R.Jimerson, T. D. Oakland y P. T. Farrell (Eds.), The Hanbook of International School Psychology (pp.103-111). London, U.K.: Sage Publications, Inc.

Newcombe, N. S. (2000). Biology is to Medicine as Psychology is to Education: True or False? En D. F. Halpern y M. D. Hakel (Eds.). Applying the Science of Learning to University Teaching and Beyond, New Directions for Teaching and Learning (pp. 9-18). San Francisco, CA: JosseyBass.

SEC (2007). Towards more knowledge-based policy and practice in education and training" .1098, p 56. (http://ec.europa.eu/education/policies/2010/ doc/sec1098_en.pdf).

Seifried, K. (2008). Schulpsychologie im internationalen Vergleich, wo steht Deutschland? BDP, 2008.

Squires, G. y Farrell, P. (2007) . Educational Psycholoy in England and Wales. In S. R. Jimerson, T.D. Oakland y P.T. Farrell (Eds.), The Hanbook of International School Psychology (pp. 81-90). London, U.K.: Sage Publications, Inc.

Topping, K. J., Smith, E., Barrow, W., Hannah, E. y Kerr, C. (2007). Professional Educational Psychology in Scoland. . In S. R.Jimerson, T. D. Oakland \& P. T. Farrell (Eds.), The Hanbook of International School Psychology (pp.339-350). London, U.K.: Sage Publications, Inc.

Wooldridge, A. (1994), Measuring the mind: Education and psychology in England c1860c1990. Cambridge: Cambridge University Press.

Sipilä-Lähderkorpi, P. (2004). Selvitys koulukuraattori ja psykologitoiminnan järjestämisestä kunnissa 1990-1992. Sosiaali-ja terveyshallitus. Tiedote Newsletter, 1.

Manuscrito recibido: 14/12/2010 Revisión recibida: 20/01/2011 Manuscrito aceptado: 24/01/2011 